\title{
FIFTY-FIFTH REGULAR MEETING OF THE SAN FRANCISCO SECTION
}

The fifty-fifth regular meeting of the San Francisco Section of the Society was held at Reed College on Saturday, June 2, 1928. In the absence of Chairman Bell, Professor E. E. DeCov acted as temporary chairman. The total attendance was 26, including the following 19 members of the Society:

J. R. Ballantine, Bernstein, Butler, J. W. Campbell, A. F. Carpenter, D. R. Davis, Donahue, Gavett, Griffin, Hanawalt, Jerbert, Kent, Langford, McAlister, W. E. Milne, Moritz, Mullemeister, Neikirk, Smail.

The Secretary announced that, by invitation of the Program Committee, Professor E. T. Bell will address the Section at its October meeting on the topic The theory of algebraic numbers in the light of Kronecker's program.

On motion of Professor Moritz, it was resolved: That the Section express its sense of loss in the death on May 29 of Professor R. E. Allardice, one of the organizers, a former Chairman, and a contributor to the program of the Section.

The Secretary announced that Chairman Bell appointed the following committee to nominate officers at the October meeting of the Section: Bernstein, Griffin, E. R. Hedrick.

Professor Kent invited the Section to hold its next Summer meeting at the Oregon State Agricultural College. The invitation was accepted.

Following the meeting, the members and their friends were greeted by President Colman of Reed College at a luncheon given them by the College. A drive up the beautiful Columbia River Highway followed the luncheon.

Titles and abstracts of the papers presented at the meeting follow below. The papers by Ayres, Neikirk, Roberts, and Whyburn were read by title. Miss Haycraft and Miss Nelson were introduced by Professor Griffin. Mrs. Williamson was introduced by Professor Carpenter. 


\section{Professor B. A. Bernstein: Irredundant sets of postulates} for the logic of propositions.

Alonzo Church has defined a set of postulates to be irredundant if the postulates are independent and no postulate can be "weakened" with respect to the set (Transactions of this Society, vol. 27, p. 318). In the present paper the author gives a number of irredundant sets of postulates for the logic of propositions (see Transactions of this Society, vol. 28, p. 472). The sets are free from the objections usually to be raised against a set of postulates made irredundant by Church's "mechanical" method.

2. Professor J. W. Campbell: Catenary problems in mechanics.

The solutions of catenary problems in mechanics are greatly facilitated when in addition to the functions $\sinh x, \cosh x$, and $\tanh x$ there are available tables of values of $(\sinh x) / x,(\cosh ) x-1)) / x$, and $(\cosh x) / x$. Tables of these functions from $x=0.000$ to $x=3.000$ have been compiled and tabulated by the writer and their use in connection with various types of catenary problems shown.

\section{Professor A. F. Carpenter: Tetrads of ruled surfaces.}

In this paper the projective differential properties of four ruled surfaces whose line elements are in correspondence in sets of four, are determined by means of the invariants and covariants of a system of eight ordinary linear homogeneous differential equations of the first order in eight dependent variables, to which are adjoined four linear homogeneous relations between these variables.

4. Professor F. L. Griffin: Points of minimum travel for a distributed population, II.

In this paper Professor Griffin discusses further cases of the problem considered at the Vancouver meeting of the Section.

5. Professor F. L. Griffin: Note on an investment problem involving mean values and probability.

This note calls attention to an interesting elementary illustration of mean values and probability which occurs in connection with long-term leases containing optional building clauses and accompanying adjustment of rentals, when it is a question of purchasing the lessor's rights at a price to yield a specified rate of interest.

\section{Miss Alace Haycraft: Concerning geodesics on certain} surfaces of revolution.

Miss Haycraft studies in some detail the geodesics on the circular paraboloid and hyperboloid of one sheet, and she contrasts these geodesics with corresponding curves on the cylinder, cone, and sphere. 


\section{Dr. A. R. Jerbert: Quadruples of space curves.}

A system of four linear, homogeneous, differential equations of the first order is interpreted as defining, to within a projectivity, four space curves. By virtue of their expression in terms of a common parameter, these curves are in one-to-one point correspondence. If corresponding points are joined by straight lines, a tetrahedron is formed. The study of the four associated curves is accordingly equivalent to the study of a oneparameter family of tetrahedrons. The latter point of view derives some interest from the fact that the tetrahedron configuration occurs frequently in projective differential geometry.

\section{Professor W. E. Milne: The behavior of a boundary} value problem as the interval becomes infinite.

The investigation applies to the linear differential equation $d^{2} u / d x^{2}$ $+G(x, \lambda)=0$ with linear homogeneous boundary conditions at the ends of an interval $a \leqq x \leqq b$. The object is to determine the behavior of the characteristic numbers, characteristic solutions, and oscillation properties as $a$ and $b$ approach $-\infty$ and $+\infty$ respectively. It is assumed that $G(x, \lambda)$ increases from $-\infty$ to $+\infty$ as $\lambda$ increases from $-\infty$ to $+\infty$, and also that as $x$ becomes positively or negatively infinite, $G(x, \lambda)$ becomes negatively infinite. Then as the interval becomes infinite, the characteristic numbers approach a set of critical values $\lambda_{0}, \lambda_{1}, \lambda_{2}, \cdots$, which have a limit point at plus infinity only, and which are entirely independent of the boundary conditions. The corresponding characteristic solutions approach critical solutions $u_{0}, u_{1}, u_{2}, \cdots$, which are independent of the boundary conditions. The solution, $u$, vanishes exactly $n$ times in the infinite interval. Furthermore the integrals from minus infinity to plus infinity of $(d u / d x)^{2}$ and of $G(x, \lambda) u^{2}$ exist if $u$ is any critical solution, but do not exist for any other solution of the differential equation.

9. Professor R. E. Moritz: An elementary derivation of the probability function.

The view-point in the derivation is purely geometric. The procedure is the gradual deformation of the frequency polygon corresponding to binomial distribution $(p+q)^{n}$. By a proper choice of coordinates and repeated application of Stirling's formula it is shown that as $n$ is indefinitely increased the frequency polygon goes over into the curve $y=\exp \left(-\pi x^{2}\right)$.

10. Professor R. E. Moritz: A new theory of depreciation. Preliminary report.

All known methods for the determination of the depreciation of physical assets proceed on the assumption that the life time of the asset is known. In this paper the writer assumes that the repair function is known and attempts to determine the life of the asset so as to make the annual charge to production a minimum. 
11. Professor L. I. Neikirk: A class of totally discontinuous functions. Second paper.

This paper contains a generalization of the results of the first paper presented to the Section last Spring. The problem is to construct a function $u(x)$ with the following properties: it is equal to some one of the functions $f(x), f_{1}(x), f_{2}(x), \cdots, f_{r}(x)$ at every point of the interval $(0,1)$, so that if $u\left(a_{1}\right)=f\left(a_{1}\right)$ and $u\left(a_{2}\right)=f\left(a_{2}\right)$, where $0 \leqq a_{1}<a_{2} \leqq 1$, then at some number, $a^{\prime}$, where $a_{1}<a^{\prime}<a_{2}, u\left(a^{\prime}\right)=f_{i}\left(a^{\prime}\right), 1 \leqq i \leqq r$; and if $u\left(b_{1}\right)=f_{i}\left(b_{1}\right)$ and $u\left(b_{2}\right)=f_{i}\left(b_{2}\right)$, where $0 \leqq b_{1}<b_{2} \leqq 1$, then there is a $b^{\prime}, b_{1}<b^{\prime}<b_{2}$, at which $u\left(b^{\prime}\right)=f\left(b^{\prime}\right)$; and also if $u\left(c_{1}\right)=f_{2}\left(c_{1}\right)$ and $u\left(c_{2}\right)=f_{2}\left(c_{2}\right)$, where $0 \leqq c_{1}<c_{2} \leqq 1$, then there are numbers $c_{s}^{\prime}, s=1,2, \cdots, i-1, i+1, \cdots, r$ and $c_{1}<c_{s}^{\prime}<c_{2}$ such that $u\left(c_{s}^{\prime}\right)=f_{s}\left(c_{s}^{\prime}\right)$. The function $u(x)$ is constructed and is defined explicitly.

\section{Miss Mildred Nelson: A further generalization of the} circular and hyperbolic functions of sectorial areas.

The generalization of circular and hyperbolic functions presented by Miss Pennock at the Vancouver meeting of the Section is extended by Miss Nelson. She substitutes an ellipse for the circle and an hyperbola of unequal semi-axes for the usual rectangular hyperbola; also she considers se:tional areas from points asymmetrically located as well as from points on the transverse axis.

13. Mr. J. H. Roberts and Mr. J. L. Dorroh: On a problem by G. T. Whyburn.

In his recent paper Concerning irreducible cuttings of continua, G. T. Whyburn gives an example of a continuum $M$ which contains no indecomposable continuum, but which contains two points $A$ and $B$, such that no cutting of $M$ between $A$ and $B$ is irreducible. He proves that if $M$ is an indecomposable continuum, then every two points $A$ and $B$ of $M$ are such that no irreducible cutting of $M$ between $A$ and $B$ exists. He raises the following questions: (1) If a continuum $M$ is such that no subset of $M$ is an irreducible cutting of $M$ between any two points $A$ and $B$ of $M$, then is $M r$ necessarily indecomposable? (2) If every cutting of a continuum $M$ is reducible, is $M$ necessarily indecomposable? In the present paper it is shown by an example that the answer to both of these questions is in the negative. Also, it is proved that a necessary and sufficient condition that every subcontinuum of a continuum $M$ be indecomposable is that no subcontinuum of $M$ contain an irreducible cutting of itself.

14. Mr. J. H. Roberts: Concerning closed sets which contain no domain in n-dimensional euclidean space.

The author has recently shown that if $M$ denotes a plane continuum containing no domain, then there exists a continuous one-to-one transformation of the plane into itself such that in the transformed plane there exists a rectangular coordinate system with the property that if $l$ denotes 
any line parallel to one of the coordinate axes, then the set of points common to $l$ and $M$ is either vacuous or totally disconnected. This result is included in the following more general theorem, proved in the present paper: If $S$ is an $n$-dimensional euclidean space, and $M$ is a closed subset of $S$ containing no $n$-dimensional domain, then there exists a continuous one-to-one transformation of $S$ into itself, such that in the transformed space every straight line contains at most a totally disconnected subset of $M$.

\section{Professor L. L. Smail: On the effect of the operation of} summability on the interval of oscillation of an infinite sequence. Preliminary report.

Practically all previous studies of the summability of divergent series have been concerned with the unique limit of an auxiliary sequence associated with the given series. This paper treats of the relation between the upper and lower limits of the auxiliary sequence whose unique limit, when it exists, gives the usual generalized sum of the given sequence. This relation is investigated for the general methods of summability, and also for the Cesàro method when the order of summability is increased.

16. Professor G. T. Whyburn: Concerning cuttings of continua.

In this paper it is shown that in a locally compact metric and separable space: (1) no continuum $M$ contains an uncountable collection of mutually exclusive connected subsets each containing a compact proper set which cuts $M$; (2) the author's theorem (previously established in the plane) that all save a countable number of the cut points of any continuum $M$ are points of order two (Menger-Urysohn) of $M$ holds true; and indeed that if $G$ is any collection of mutually exclusive connected cuttings of $M$, then all save a countable number of elements of $G$ can be $\epsilon$-separated by two sets of $G$; (3) all save a countable number of the cut points im kleinen of any continuous curve $M$ are points of order two of $M$; (4) the junction points of every continuous curve are countable; and (5) if every subcontinuum of a continuous curve $M$ contains uncountably many cut points im kleinen of $M$, then $M$ is a Menger regular curve. Also the notion of a junction continuum, an extension of the notion of a junction point due to R. L. Moore, is discussed, and it is pointed out that no plane continuum contains an uncountable collection of mutually exclusive junction continua of itself.

17. Professor E. H. Mc Alister: On the dynamical form of Hooke's law. Preliminary report.

Hooke's law leads to results not in agreement with experiment when the velocity of strain is not negligible. In particular, the rapid decay of elastic vibrations finds no explanation in Hooke's law, and is accounted for only in small part by the resistance of the air. Kelvin conjectured some internal resistance, and coined the phrase "viscosity of metals," but concluded that the resistance is not merely proportional to the velocity, as in viscous 
fluids. This paper attempts to formulate a law suitable for dynamical conditions, as Hooke's law is for statical conditions. The point of mathematical interest is the search for an unknown function by mathematical means, guided by experiment. A form is found which agrees with certain experiments on torsional vibrations of a steel wire. The mathematical investigation indicates that other forms are possible, and the results of this paper are merely tentative, awaiting further research. The paper is therefore presented as a preliminary report. The tentative law admits of isochronous vibrations, in accordance with Rayleigh's dictum, and reduces to Hooke's law when the velocity is null or negligible.

18. Professor D. R. Davis: The inverse problem of the calculus of variations in space of $(n+1)$ dimensions.

In this paper is considered a system of $n$ differential equations of the form $H_{j}\left(x, y_{i}(x), y_{i}{ }^{\prime}(x), y_{i}^{\prime \prime}(x)\right)=0,(i, j=1, \cdots, n)$, whose solutions are $y_{i}=y_{i}(x)$, with the derivatives $y_{i}^{\prime}, y_{i}^{\prime \prime}$. The conditions required for the given system to have equations of variation which are self-adjoint are found. It is then proved that these conditions are sufficient to insure that a function $f\left(x, y_{1}, \cdots, y_{n}, y_{1}^{\prime}, \cdots, y_{n}^{\prime}\right)$ can be determined such that the given equations are the differential equations of the solutions for the problem of minimizing the integral

$$
I=\int_{x_{1}}^{x_{2}} f\left(x, y_{1}, \cdots y_{n}, y_{1}^{\prime}, \cdots, y_{n}^{\prime}\right) d x
$$

Incidentally, in this paper, are also found necessary and sufficient conditions that there exist a solution for a system of linear partial differential equations of the first order of the form $B_{i y_{i}}-B_{i y_{j}}=\phi_{i j}, B_{i x}-A_{y_{i}}=\phi_{i}$, $(i, j=1, \cdots, n)$, where $A, B_{1}, \cdots, B_{n}, \phi_{i j}, \phi_{i}$ are functions of $x, y_{1}, \cdots, y_{n}$, and $\phi_{i j}=-\phi_{j i}$.

\section{Professor G. T. Whyburn: Concerning certain points} of continuous curves.

In this paper a study is made of the im kleinen cut points, im kleinen cycle points, and ramification points (i.e., points of Menger order $>2$ ) of a bounded continuous curve $M$ in $n$ dimensions. The point $P$ of $M$ is called an im kleinen cycle point of $M$ if for each $\epsilon>0, M$ contains a simple closed curve of diameter $<\epsilon$ containing $P$. Some of the results proved are as follows. Let $K, N, H$, and $W$ denote the set of all im kleinen cut points, im kleinen cycle points, end points, and ramification points respectively of $M$. Then (1) $K+H+N=M$, (2) $K \cdot N$ and $K \cdot W$ are countable, (3) $K$. $N \subset W,(4)$ if $W$ is dense on an arc $t$ of $M$, both $N$ and $M-K$ are uncountably dense on $t,(5)$ if $M$ is cyclicly connected and $M-K$ is not uncountably dense on any subcontinuum of $M$, then (a) $W$ is totally disconnected, and (b) every component of $M-W$ is an arc segment, (6) if $M=K$, then for each $\epsilon>0, M$ is the sum of a finite number of $\epsilon$-continua no two having more than one common point. 


\section{Professor G. T. Whyburn: Concerning a certain type of regular curve.}

A continuous curve $M$ will be called a node curve provided that for each $\epsilon>0, M$ is the sum of a finite number of $\epsilon$-continua each having at most two points in common with the rest of $M$. In this paper a study is made of node curves, and some of the results found are as follows. Let $M$ denote any bounded continuous curve in $n$ dimensions. Then (1) $M$ is a node curve if and only if it is true that if $C$ is any maximal cyclic curve of $M$ then (a) $C$ is a node curve and (b) every point of $C$ which is a limit point of some component of $M-C$ is an im kleinen cut point of $C$; (2) if $M$ is cyclicly connected, $M$ is a node curve without points of order $w$ if and only if no ramification point of $M$ is an im kleinen cycle point of $M$; (3) if no point of $M$ is an im kleinen cycle point, then $M$ is a node curve; (4) the ramification points of a node curve are countable and each of them is an im kleinen cut point.

\section{Professor G. T. Whyburn: A continuous curve in} 3-space.

In this paper a simple example is given in 3 dimensions of a continuous curve $\psi$ every subcontinuum of which is a continuous curve which has the following properties: (1) $\psi$ contains two points $A$ and $B$ and infinitely many $\operatorname{arcs} A X_{i} B(i=1,2,3, \cdots)$ from $A$ to $B$ no two of which have any common points except $A$ and $B$, (2) $\psi$ contains an arcwise connected subset which is not arcwise connected im kleinen (in fact, not connected im kleinen at all) and which has an inaccessible boundary point, and (3) $\psi$ contains a connected subset which is not arcwise connected. It is already known that $\psi$ could not lie in the plane and have properties (1) or (2). It is also pointed out in this paper that no Menger regular curve can have properties (1) or (2), and a number of related questions are settled in $n$ dimensions for regular curves and for continuous curves all of whose subcontinua are continuous curves.

22. Mrs. E. N. Williamson: Two plane curves in point correspondence.

This paper deals with the projective differential properties of two plane curves in one-to-one reciprocal point correspondence, making use of a defining system of three ordinary linear homogeneous differential equations in two dependent variables, two of the equations being of the second order and one of the first. Geometric interpretations are obtained for certain of the invariants and covariants of the system.

23. Dr. L. Ayres, (National Research Fellow): Concerning the arc-curves and the basic sets of a continuous curve. Second Paper.

This paper studies the relations between the cyclic elements of a continuous curve and the arc-curves, and develops some new properties of the 
basic sets of a continuous curve. It is shown that every arc-curve of more than one point is a collection of cyclic elements, and every connected collection of cyclic elements is an arc-curve. Every arc-curve of two points is a simple cyclic chain and conversely. A subset' $K$ of a continuous curve $M$, which is not cyclicly connected, is an irreducible basic set of $M$ if and only if (1) no point of $K$ is a cut point of $M$, (2) every point of $K$ belongs to some node of $M$ and each node of $M$ contains exactly one point of $K$. An irreducible basic set of a continuous curve $M$ contains the set of all end points of $M$, and an irreducible basic set has many of the properties of the set of all end points.

B. A. Bernstein.

Secretary of the Section 